\title{
Fluid dynamic mechanisms of enhanced power generation by closely spaced vertical axis wind turbines
}

\author{
Stefania Zanforlin ${ }^{1,}{ }^{*}$ and Takafumi Nishino ${ }^{2}$
}

${ }^{1}$ Department of Energy, Systems, Territory and Constructions Engineering, University of Pisa, 1.go Lucio Lazzarino, 56122 Pisa, Italy.

${ }^{2}$ Centre for Offshore Renewable Energy Engineering, Cranfield University, Cranfield, Bedfordshire MK43 OAL, UK.

* Corresponding author. Tel.: +39-050-2217145; fax: +39-050-2217150; e-mail address:

s.zanforlin@ing.unipi.it.

\section{Abstract}

We present a comprehensive set of two-dimensional (2D) unsteady Reynolds-averaged Navier-Stokes (URANS) simulations of flow around a pair of counter-rotating vertical-axis wind turbines (VAWTs). The simulations are performed for two possible configurations of the counter-rotating VAWT pair, with various gaps between the two turbines, tip-speed-ratios and wind directions, in order to identify key flow mechanisms contributing to the enhanced performance of a pair of turbines compared to an isolated turbine. One of the key mechanisms identified, for the case of two turbines arrayed side-by-side with respect to the incoming wind, is the change of lateral velocity in the upwind path of each turbine due to the presence of the neighbouring turbine, making the direction of local flow approaching the turbine blade more favourable to generate lift and torque. The results also show that the total power of a staggered pair of turbines cannot surpass that of a side-by-side pair of turbines. Some implications of the present results for the prediction of the performance of single and multiple rows (or a farm) of VAWTs are also discussed. The local flow mechanisms identified in the present study are expected to be of great importance when the size of the farm is relatively small.

Key words: Counter-rotating VAWTs; Wind farm; Induced velocity; Blockage effect; Wake effect.

\section{Introduction}

Following the rapid development of onshore and offshore wind farms in recent years, there is increasing interest in how to improve the overall performance of multiple wind turbines. Whilst a number of studies on horizontal-axis wind turbines (HAWT's) have shown the importance of spacing between the turbines (as well as the array configuration) to minimise the wake loss, recent studies on a closely spaced array of vertical-axis wind turbines (VAWT's) by Dabiri and his team [1-3] have shown the possibility of achieving a much higher power density (i.e., power per unit farm area) compared to existing wind farms 
employing HAWT's. During their field measurements in Southern California in 2010 and 2011, Dabiri and his team $[2,3]$ tested various configurations of pairs of counter-rotating VAWT's closely spaced from each other, inspired by the hydrodynamic mechanism of "fish schooling" minimising the wake loss. The performance of pairs of counter-rotating VAWT's has also been investigated numerically by Feng et al. [4] using a free vortex method with empirical wake models. More recently, Araya et al. [5] has proposed a loworder model of two-dimensional flow past pairs of VAWT's using the concept of a leaky Rankine body, showing the existence of two competing fluid dynamic mechanisms (namely the local acceleration of the flow and local deceleration of the flow) that contribute to the overall array performance.

The exact mechanisms of the enhanced power generation by closely spaced pairs of VAWT's, however, are still unclear since these previous studies have not revealed detailed local flow characteristics around each turbine sufficiently. Hence in this study, we perform a comprehensive set of two-dimensional unsteady Reynolds-averaged Navier-Stokes (URANS) simulations of a single and a pair of counter-rotating VAWT's, to compare detailed local flow characteristics around the turbine blades and thereby identify key fluid dynamic mechanisms that explain the increased performance of a pair of turbines relative to an isolated turbine. The simulations are performed for two possible configurations of the counter-rotating turbine pair, with various gaps between the two turbines, tip-speed-ratios and wind directions. The results show clearly how, and why, the values of torque generated during the upwind path and downwind path of each turbine are affected by the presence of the neighbouring turbine. Although this study is concerned with vertical-axis wind turbines, the majority of the findings and conclusions obtained in this study are applicable to verticalaxis tidal/marine turbines as well.

It should be noted that a number of Computational Fluid Dynamics (CFD) studies of a vertical-axis turbine using 2D URANS simulations have already been reported in the past. A recent extensive review of these CFD studies can be found in [6]. A general consensus from these earlier CFD studies is that carefully designed 2D URANS simulations are capable of predicting the influence of the turbine on the flow around 64 the turbine as well as the performance of the turbine qualitatively correctly, especially for an $\mathrm{H}$-shape 65 Darrieus turbine with a high aspect ratio (which helps minimise 3D flow effects). Nevertheless, the majority 66 of the earlier CFD studies have focused on the performance of a single turbine; investigations into the interaction of two vertical-axis turbines closely spaced from each other are still limited.

This study is based on the $1.2 \mathrm{~kW}$ Windspire VAWT [7], a commercial turbine for micro-generation. The diameter of the turbine (D) is $1.20 \mathrm{~m}$, the chord length (c) is $0.128 \mathrm{~m}$ and therefore the solidity $\left(\sigma=\mathrm{B} * \mathrm{c} /\left(\pi^{*} \mathrm{D}\right)\right.$, where $\mathrm{B}=3$ is the blade number) is 0.10 , which is typical for medium-high solidity VAWTs for urban areas. We chose this turbine for three reasons. The first reason is the availability of experimental data taken by the manufacturer in an open field, which avoids the need to correct wind tunnel data by taking into account blockage effects. The second reason is its large aspect ratio (the ratio of blade length to turbine diameter is 5) that reduces the influence of 3D aerodynamics (associated with blade tip losses), allowing a comparison of 2D CFD results with the experimental data. The third reason is the possibility of a comparison 
numerical campaign carried out by Dabiri and his team [1-3] and, more recently, in the numerical analysis by

Feng et al. [4].

\section{Model set-up}

We start by constructing a computational domain using the commercial mesh generator ANSYS ICEM. The size of the computational domain is 57D (35D in upstream, 22D in downstream) along the $\mathrm{x}-$ coordinate, and 100D along the $\mathrm{y}$-coordinate, where $\mathrm{D}$ is the turbine diameter. The positions of inlet and lateral boundaries are far enough for the flow to be considered unbounded, i.e., the boundaries have negligible influence on the characteristics of the flow oncoming the turbine. The position of the outlet boundary allows a complete wake development.

Two different grid levels are adopted: a fixed sub-grid with the external dimensions of the flow domain, and one (or two, in case of a turbine pair) rotating sub-grid that includes the VAWT geometry and allows a relative motion with respect to the fixed grid. This grid arrangement utilises the sliding mesh technique [8] and allows the simulation of the rotational motion of the turbine with an unsteady Reynoldsaveraged Navier-Stokes (URANS) analysis. The grids are everywhere unstructured with the exception of the region around the blades, where 14 structured layers of quad elements are set to better predict the boundary layer phenomena. The grids are finer near the blade surface (and in particular where flow separation occurs due to dynamic stall) and progressively coarser outward. As shown in figure 1 a high density grid is also set in the near wake region and far downstream to accurately simulate the wake development and any aerodynamic interferences between the wakes of a turbine pair.
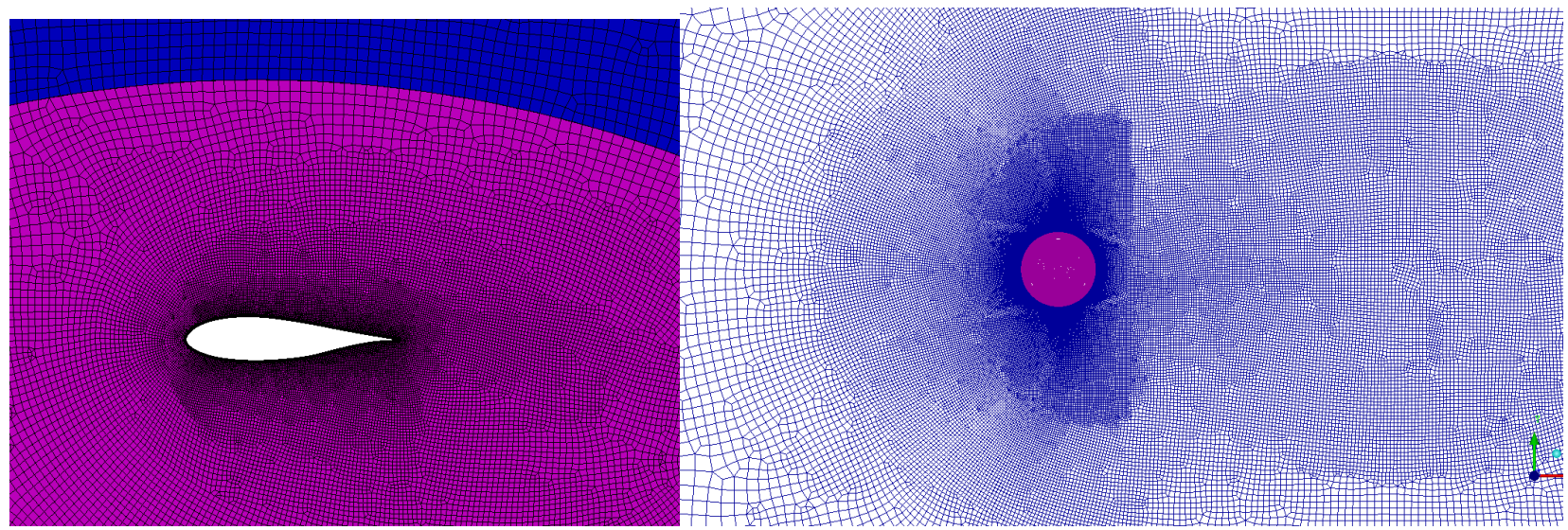

FIGURE 1: (left) details of the grid around the blades and (right) in the near/far wake regions (only a part of the whole domain is shown); different colours indicate the rotating and the fixed sub-grids.

The number of grid points around the airfoil profile (suction plus pressure sides) is 440 . The wall distance from the first layer of cells is set at $2.3 * 10^{-4} \mathrm{c}$, where $\mathrm{c}$ is the blade chord length, resulting in the maximum $\mathrm{y}^{+}$ (dimensionless wall distance) of less than 3 (except for the trailing edge region, where $\mathrm{y}^{+}<5$ ). The rotating sub-grid consists of $\sim 120,000$ elements (for each one, in case of a turbine pair); the fixed sub-grid consists of $\sim 130,000$ and $\sim 150,000$ elements for a single turbine and a pair of turbines, respectively. Across the inlet, the Dirichlet boundary condition is specified with a uniform velocity $\mathrm{U}_{0}$ of $8.0 \mathrm{~ms}^{-1}$. According to typical built 
environments, the turbulence intensity and length scale are set to $4 \%$ and $1 \mathrm{~m}$, respectively, at the inlet. The upper and lower boundary conditions are set to a symmetric condition. At the exit boundary, a fixed pressure equal to the free stream condition is specified.

Computations were performed using the commercial CFD solver ANSYS FLUENT v.15, using its "pressure-based" segregated solver for the URANS equations. Turbulence is modelled using the k- $\omega$ SST (Shear Stress Transport) model. The principle behind the SST model is the combination of two different turbulence models: the $\mathrm{k}-\omega$ model in the inner part of the boundary layer, and the $\mathrm{k}-\varepsilon$ model in the freestream. This turbulence scheme was adopted because of its aptitude in cases involving high adverse pressure gradients and therefore smooth surface separations [9]; it has proved to be particularly efficient for VAWTs due to its ability to simulate more accurately the vortices that are seen during dynamic stall at low TSR than the $\mathrm{k}-\omega$ and $\mathrm{k}-\varepsilon$ models [10]. The air is considered as incompressible since the operating conditions do not exceed a local Mach number greater than 0.3. The settings for the simulations are shown in table 1 . The convergence criteria is set at $1 * 10^{-4}$ for all residuals. Thirty turbine revolutions are simulated: for the first 20 rev. a coarse time-step corresponding to $2^{\circ}$ azimuthal angle of turbine rotation is used; for the successive 10 rev. a finer time-step corresponding to $0.5^{\circ}$ azimuthal angle is used.

\begin{tabular}{|c|c|}
\hline \multicolumn{2}{|c|}{ Solver } \\
\hline Type & Pressure-based \\
\hline Time & Transient \\
\hline \multicolumn{2}{|c|}{ Solution methods } \\
\hline Pressure-Velocity coupling & PISO \\
\hline \multicolumn{2}{|c|}{ Spatial discretization } \\
\hline Gradient & Least squares cell based \\
\hline Pressure & PRESTO! \\
\hline Momentum & Second order upwind \\
\hline Turbulent kinetic energy & Second order upwind \\
\hline Specific dissipation rate & Second order upwind \\
\hline \multicolumn{2}{|c|}{ Transient formulation } \\
\hline \multicolumn{2}{|c|}{ Second order implicit } \\
\hline
\end{tabular}

TABLE 1: Settings for the CFD simulations

\section{Model validation}

The blade profile of the $1.2 \mathrm{~kW}$ Windspire VAWT is an asymmetric airfoil DU06W200, designed at the Delft University of Technology by adding $2 \%$ of thickness and a cambering of $0.8 \%$ to the symmetric NACA0018 profile. Experimental force coefficients can be found in the thesis work of Claessens [11]. The turbine operates with variable angular velocity, $\Omega$, by means of an electronic control system that allows to maintain the tip speed ratio ( $T S R=R * \Omega / U_{0}$, where $\mathrm{R}=0.6 \mathrm{~m}$ is the turbine radius) at an optimal value of 2.3 and the power coefficient $\left(\mathrm{C}_{\mathrm{P}}=\mathrm{P} /\left(0.5^{*} \rho^{*} \mathrm{U}_{0}{ }^{3 *} \mathrm{D}\right)\right.$, where $\mathrm{P}$ is the power per meter of blade, and $\rho$ is the air density) at approximately 0.22 . The load is controlled by passive stall: for wind speed lower than $10.6 \mathrm{~m} / \mathrm{s}$ (the rated wind speed) the TSR is kept to 2.3, but for higher wind speeds the turbine speed is kept constant 
and thus the TSR decreases leading to stall. The relatively high solidity and the small size of the turbine justify the low values of both $\mathrm{C}_{\mathrm{P}}$ and the optimal TSR. In fact the operational average Reynolds number $\left(\operatorname{Re}=c^{*} R * \Omega / v\right.$, where $v$ is the kinematic viscosity) is very low $\left(\sim 160,000\right.$ for $\left.U_{0}=8 \mathrm{~m} / \mathrm{s}\right)$, entailing considerable flow separation phenomena induced by the high adverse pressure gradient occurring on the blade suction side (as already discussed in a previous study, [12]).

We performed CFD simulations of an isolated turbine first to verify the numerical model by comparing results with experimental data. Figure 2 shows a comparison between the calculated and the experimental values of power and $C_{P}$ versus the wind speed measured at the hub height. Except for very low wind speeds (that imply extremely low Reynolds numbers) and very high wind speeds (that involve stall), the numerical results compare well with the measured data; the differences are less than $20 \%$, which is reasonable considering that the experimental power is the electrical one and the CFD model includes neither the interferences of shaft and struts nor the blade tip losses. Some additional simulations were made to verify the grid sensitivity, as reported in the Appendix.
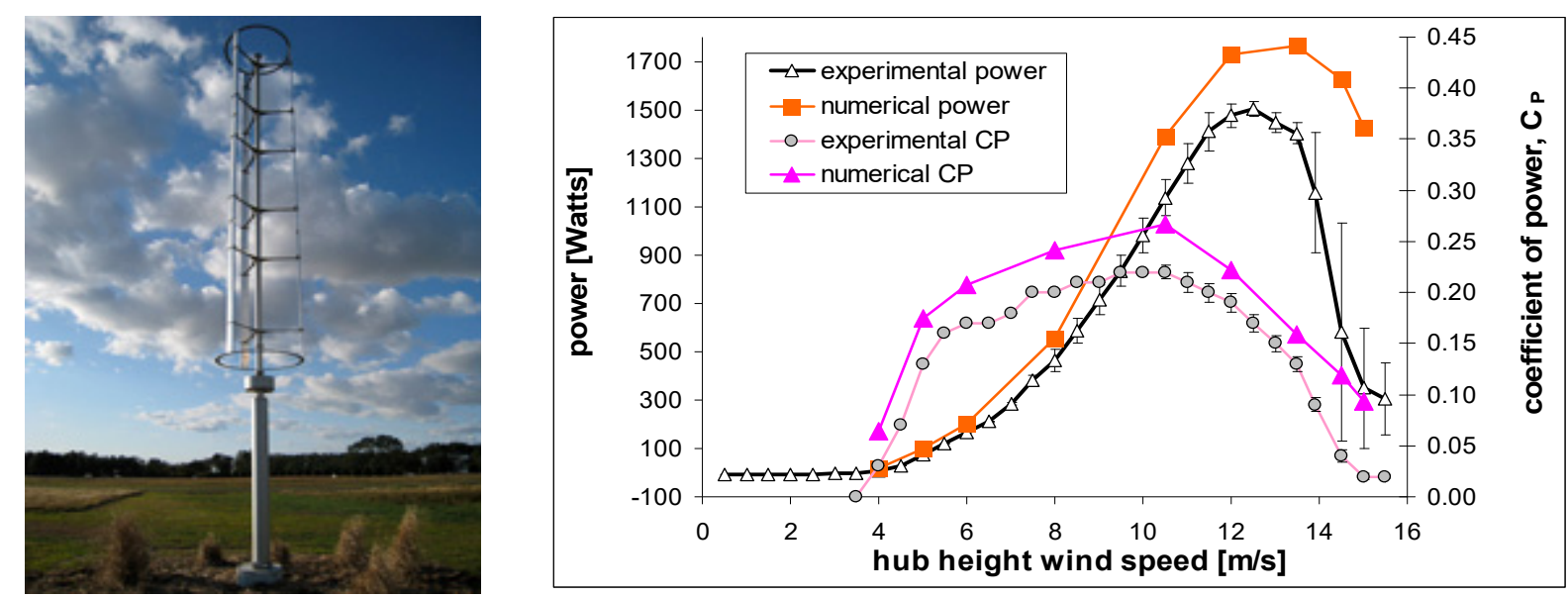

FIGURE 2: (left) Windspire 1.2kW VAWT; (right) comparison between experimental performance [7] and predictions obtained for the Windspire turbine with ANSYS Fluent CFD software.

\section{Physical mechanisms of a pair of turbines}

We analyse the behaviour of a pair of counter-rotating VAWTs in close proximity by means of 2D CFD simulations. Two possible configurations "A" and "B" (see the schematic on figure 3 for the layout definitions) are considered. All the simulations are performed for a wind speed of $8.0 \mathrm{~m} / \mathrm{s}$. Unless otherwise specified, the distance between the two turbine axes is set at $1.5 \mathrm{D}$ and TSR is set at 2.7 , which is the TSR giving the highest power for the turbine pair cases.

A schematic representation of the upwind and downwind paths of the blade in one revolution is given in figure 3; as usually done, in all graphs illustrating the instantaneous $C_{\mathrm{P}}$ for a single blade, the azimuthal position $\theta=0$ corresponds to the beginning of the upwind path of the blade. It should be observed that a blade starts its upwind path from the outer side of the configuration in case of $\mathrm{A}$, and from the inner side of the configuration in case of B. In all comparative analyses of this study the isolated turbine is considered to spin anticlockwise. 


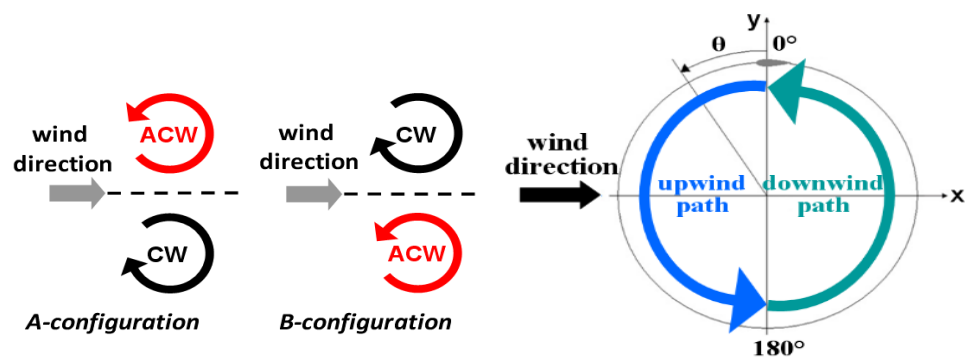

a

b

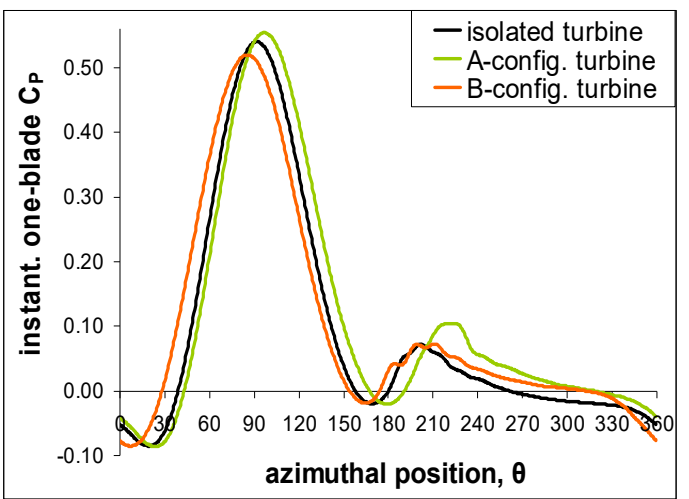

$\mathbf{C}$

FIGURE 3: (a) Definition for the layout of configurations A and B; (b) schematic representation of the upwind and downwind paths of the blade in one revolution; (c) one-blade $\mathrm{C}_{\mathrm{P}}$ during one revolution calculated for the isolated (anticlockwise) turbine and for the anticlockwise turbine in configurations $\mathrm{A}$ and $\mathrm{B}$.

Before quantitatively analysing the performance of counter-rotating VAWT pairs, we highlight some qualitative features that can be found comparing the streamlines around a pair of VAWTs to those around an isolated turbine (figure 4).

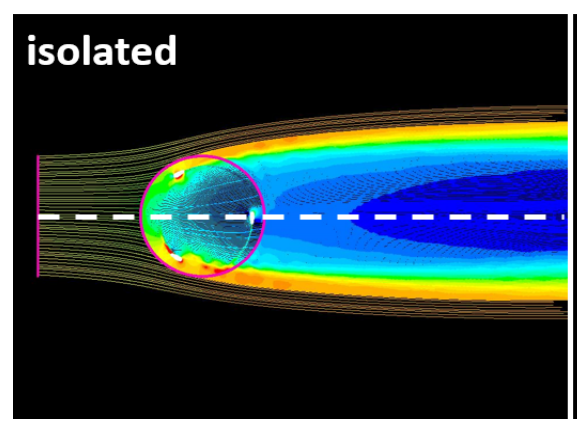

a

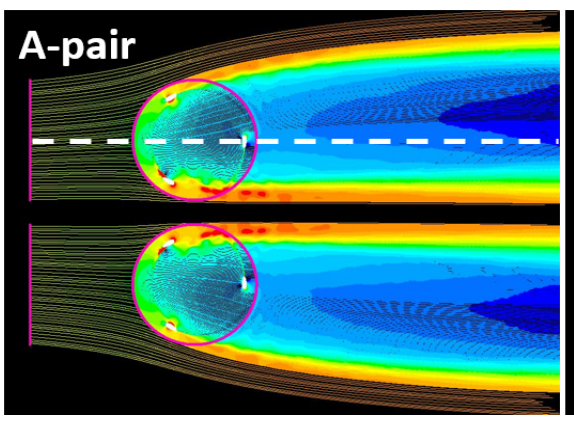

b

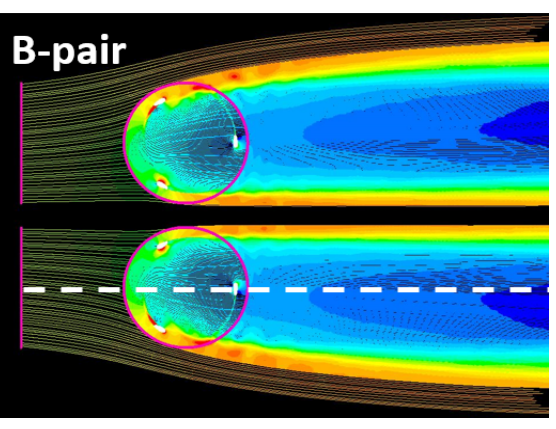

c

FIGURE 4: Streamlines coloured with velocity magnitude $[1 \div 10.5 \mathrm{~m} / \mathrm{s}]$ for the isolated turbine (a), A-pair (b) and B-pair (c); to facilitate the comparison, only the streamlines starting from grid cells intercepted by the magenta lines (the same for all the pictures) are shown; white dashed lines indicate the anticlockwise turbines.

(a) Due to rotation, an isolated turbine shows a slight wake bending; hence the wakes of Aconfiguration turbines diverge in the lateral (y) direction slightly more than the wakes of Bconfiguration turbines.

(b) Due to streamwise resistance imposed by the turbine(s), flow tends to accelerate outside of each turbine (as with an ideal actuator disc). In case of A, however, flow accelerates more significantly through the gap between the two turbines, whereas in case of $\mathrm{B}$, the flow acceleration between the two turbines is less pronounced. The difference between A and B lies in the direction of the velocity induced by the rotors (which is concordant with the wind direction for A and discordant for B). As a result, more flow tends to go outside of the two turbines for B than for A. 

parallel to the configuration symmetry plane, whereas for an isolated turbine the flow is induced to diverge at both sides.

(d) A significant wake contraction is observed at the inner sides of pair configuration (the width of the inner half of a wake appears noticeably reduced). The outer half of the wake does not change appreciably.

We examined $\mathrm{x}$ and $\mathrm{y}$-components of the flow velocity upstream the turbines (on U-curve), neardownstream (on $\mathrm{D}_{1}$-curve) and far-downstream (on $\mathrm{D}_{2}$-line). The results are plotted in figure 5 (results concerning $\mathrm{D}_{2}$-line are omitted for brevity) together with velocity magnitude maps and curve setting. The velocity values for the isolated turbine are shifted along y-coordinate to facilitate the comparison with the turbine belonging to the $\mathrm{A}$ and $\mathrm{B}$ configurations and spinning in the same rotational direction. Also, the velocities and distances have been non-dimensionalised by the velocity at the inlet, $\mathrm{U}_{0}$, and the rotor diameter, D, respectively. It should be noted that: (1) the decrease of x-velocities on U-curve suggests a reduction of the flow rate through the turbines, especially for the B-pair; (2) y-velocities are greatly reduced during the early upwind path for B and during the late upwind path for A (namely, at the inner sides of the configuration); and (3) as a consequence of the reduced flow rate through the turbines, a moderate increase of y-velocities occurs during the early upwind path for A and during the late upwind path for B (namely, at the outer sides of the configuration).

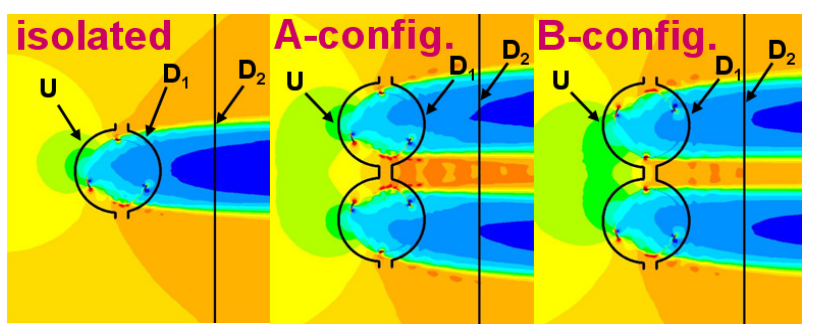

$\mathbf{a}$
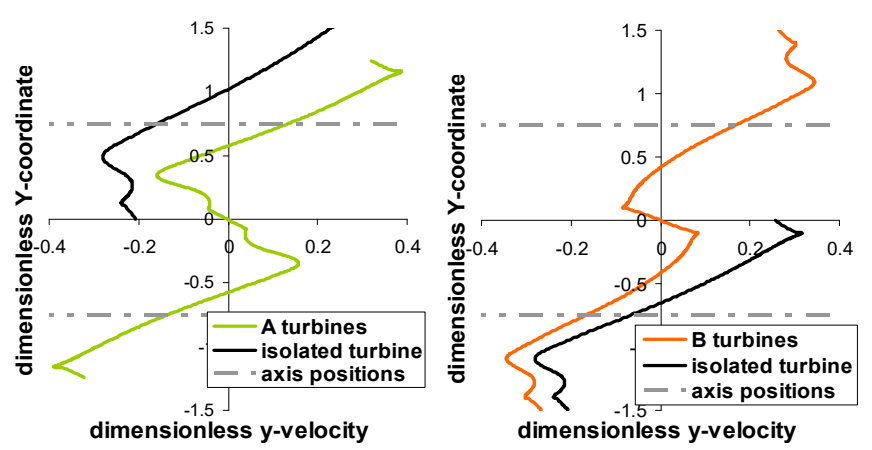

c
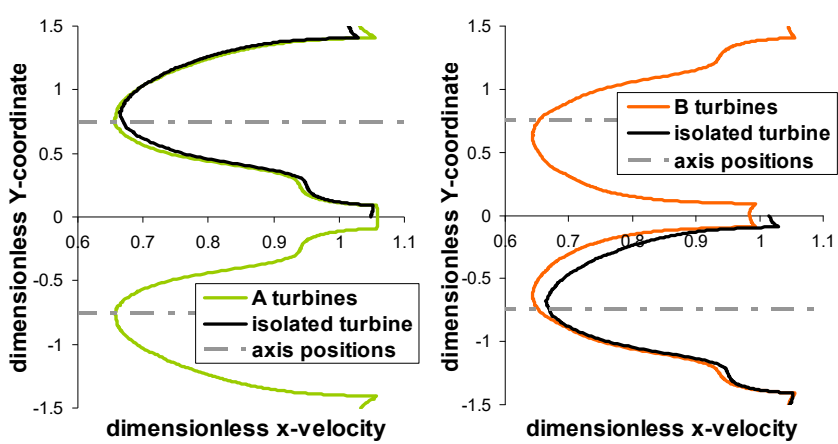

b
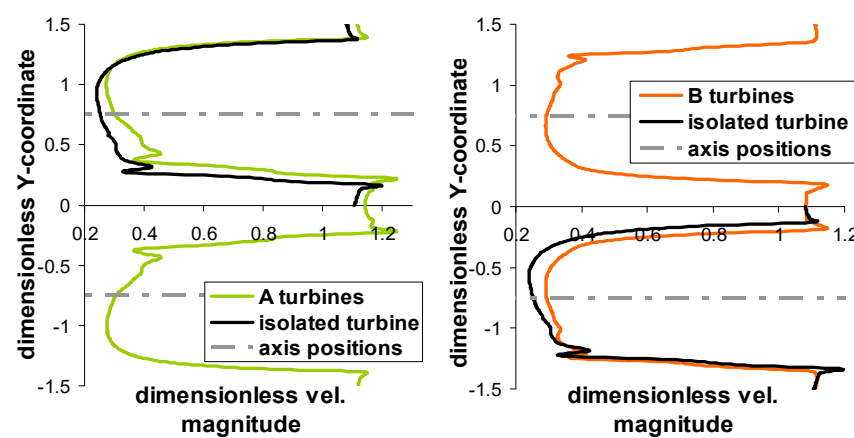

d

FIGURE 5: (a) Velocity magnitude maps $[1 \div 10.5 \mathrm{~m} / \mathrm{s}]$ for the isolated turbine and for the side-by-side A and B configurations, and the curves set to compare the velocity components; (b) dimensionless x-velocity on U-curve; (c) dimensionless y-velocity on Ucurve; (d) dimensionless velocity magnitude on $\mathrm{D}_{1}$-curve. Results refer to a particular time step of the unsteady solution (blades at $0^{\circ}$, $120^{\circ}$ and $240^{\circ}$ azimuthal degrees). 
The velocity plots in figure 5 can explain the power increase in the upwind path and in the downwind path achieved with both configurations $\mathrm{A}$ and $\mathrm{B}$ with respect to the isolated turbine, shown by the one-blade instantaneous $C_{P}$ graph in figure 3. The gain in the upwind path comes from an extension of the azimuthal range in which torque is generated; in particular the torque generation ends later for A and begins earlier for B. Importantly, this range extension is correlated to the suppression of y-velocity component (or the component diverging from the turbine axis) in the flow approaching the blade at the inner sides of configuration, as will be illustrated below.

To justify how the suppression of y-velocity in the flow approaching the blades during the upwind path can increase torque generation, we compare local flow characteristics around a blade for an isolated turbine and a turbine in B-configuration at an azimuthal position $\theta=40^{\circ}$ (beginning of the upwind path). Figure 6 depicts absolute and relative (or apparent) streamlines. It can be seen that the aerodynamic interaction between the two turbines of the B-configuration modifies the direction of the absolute flow approaching the blade and therefore the direction of the apparent flow (namely, the flow observed from the rotating blade). As a consequence, for the turbine belonging to the B-pair there is a component of lift in tangential direction (responsible for torque generation), whereas for the isolated turbine there is not.

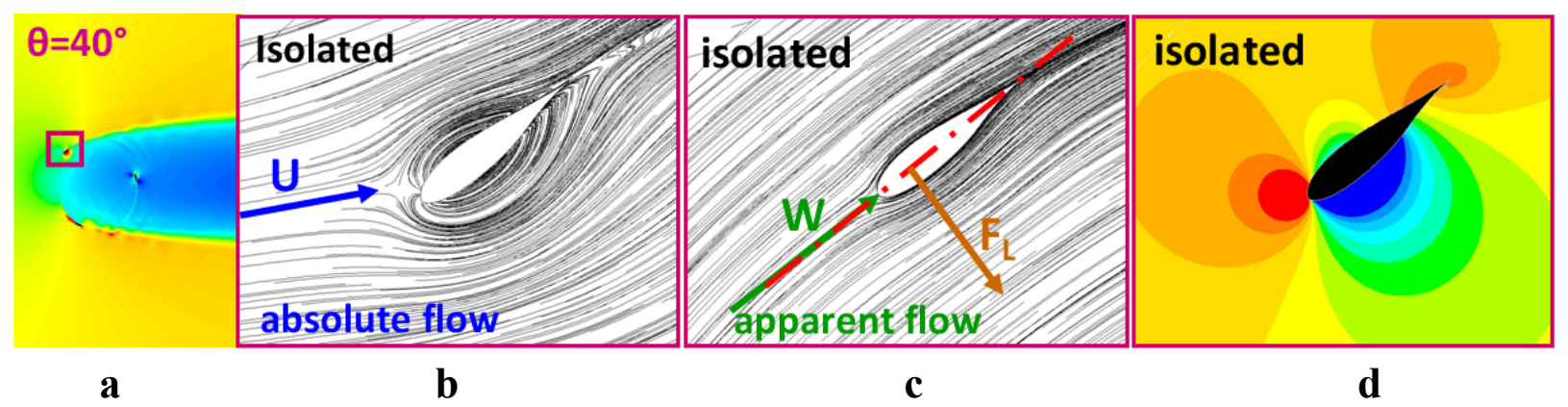

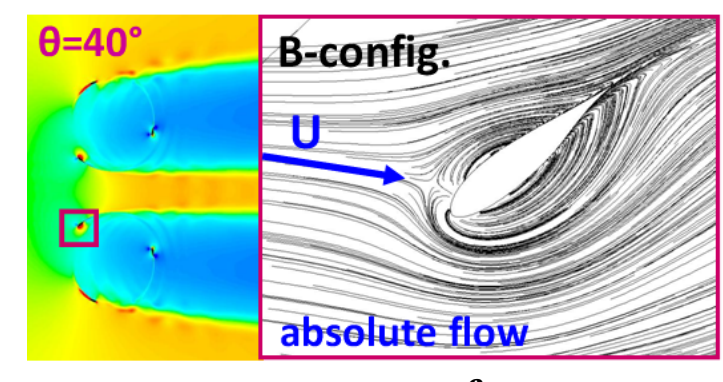

e f

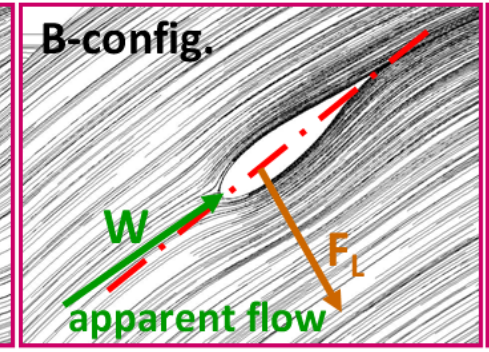

g

\section{B-config.}

FIGURE 6: Isolated turbine vs. B-configuration: (a, e) velocity magnitude maps $[1 \div 11 \mathrm{~m} / \mathrm{s}]$; (b, f) absolute and (c, g) apparent streamlines for the flow around the blade at $\theta=40^{\circ}$ (blue and green arrows indicate the direction of absolute and apparent flows, respectively; brown arrows indicate the direction of the lift force); $(\mathrm{d}, \mathrm{h})$ absolute pressure maps $[-250 \div 170 \mathrm{~Pa}]$.

The absolute pressure maps for B-configuration show a greater pressure difference between the pressure and the suction sides of the blade and therefore a higher lift, confirming the better performance achievable with a pair of counter-rotating turbines in B-configuration at $40^{\circ}$ azimuth. It should be noted that this result is obtained despite a lower flow rate (lower x-velocities) for B-configuration, demonstrating the importance of the direction of the flow approaching the blade. Qualitatively similar results were observed comparing a turbine in A-configuration with an isolated turbine during the late upwind path (not shown here for brevity). 
The power gain observed in the downwind path by both configurations with respect to the isolated turbine (see $\mathrm{C}_{\mathrm{P}}$ graph in figure 3 ) is more difficult to interpret, but it appears to be largely due to higher flow rates occurring in the near-downstream (as proved by velocity magnitude monitored on $\mathrm{D}_{1}$-curve) as a consequence of the wake contraction. This happens because at the inner sides of the configuration the flow through the downwind path is prevented to diverge laterally (as it would happen at both sides of an isolated turbine) by the presence of the second turbine, and thus it is constrained parallel to the configuration symmetry plane, accompanied by a contraction of the wake width.

We can conclude that if the turbines are aligned side-by-side, two physical mechanisms are responsible for the enhanced performance of counter-rotating VAWT pairs: (1) $y$-velocity suppression in the upwind path that makes the direction of the flow approaching the blade more favourable to generate lift and torque, and (2) wake contraction in the downwind path.

\section{Effect of staggering the two turbines}

Do these mechanisms also occur in case of staggered pairs? We investigated the behaviour of staggered $\mathrm{A}$ and $\mathrm{B}$ pairs with distances between turbine axes $\Delta \mathrm{x}=1.5 \mathrm{D}$ and $\Delta \mathrm{y}=1.5 \mathrm{D}$. Results are depicted in figures 7 and 8 . The instantaneous one-blade $C_{P}$ graphs in figure 7 show a significant performance improvement for the downstream turbine for both A and B pairs and also a (less significant) performance deterioration for the upstream turbine for the B pair.
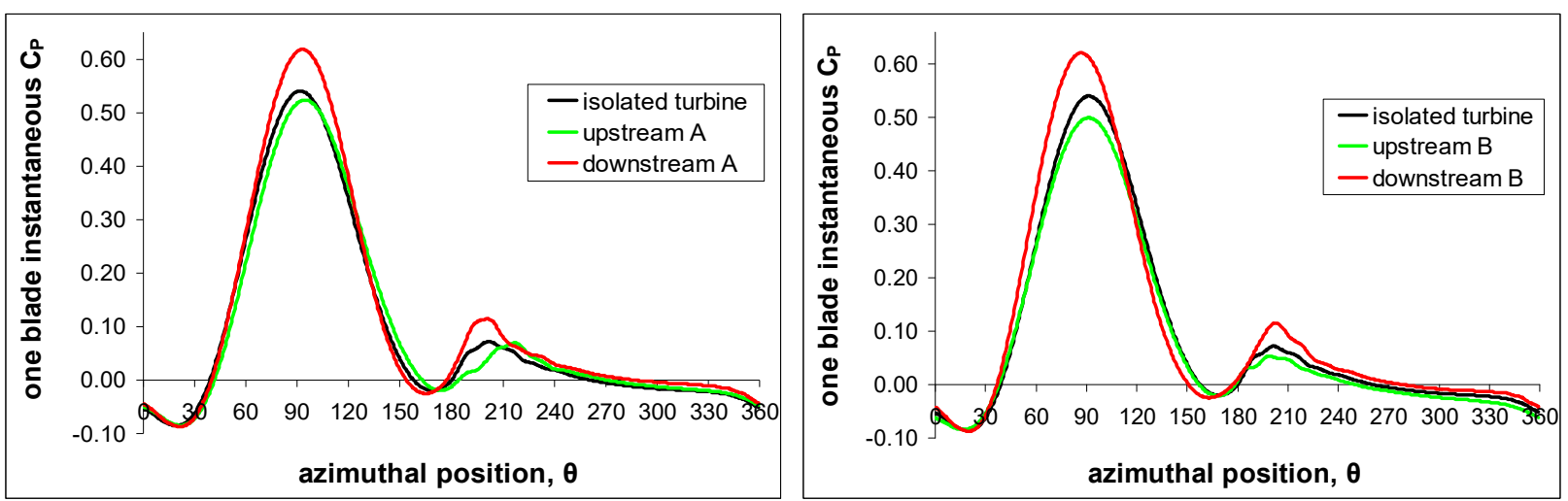

FIGURE 7: Instantaneous one-blade $\mathrm{C}_{\mathrm{P}}$ for the upstream and the downstream A turbines (left) and for the upstream and the downstream B turbines (right), compared to the isolated turbine.

The mechanism responsible for the good performance of the downstream turbine, however, is rather different from that found for side-by-side pairs. Here the dominant mechanism is an effect of the upstream turbine blockage. In particular, the high flow rate occurring at the sides of the upstream turbine contributes to the peak $C_{P}$ of the downstream turbine that is considerably higher than that of the isolated turbine (without the extension of the azimuthal range producing torque observed for the side-by-side configurations). Moreover, most of the power gain, with respect to the isolated turbine, is generated in the upwind path. Reasons for these results can be found by looking at the plots of the flow velocity monitored on $U$ and $D_{1}$ curves in figure 8 ; the values for the isolated turbine are shifted along y-coordinate and also mirrored (duplicated) to facilitate 
the comparison with the turbines spinning in the same rotational direction. X-velocities on U-curve confirm the much higher flow rate in front of the downstream turbines, whereas y-velocities are quite similar to that calculated for the isolated turbine. Velocity magnitudes on $D_{1}$ curve exhibit only a slight increase and indicate the absence of any wake contraction for the downstream turbine. There results suggest that both yvelocity suppression and wake contraction beneficial mechanisms occur only when the turbines are aligned side-by-side.

Meanwhile, the poorer performance found for the upstream B-turbine can be explained by considering the convergent wake bending, i.e. a shorter distance between the two turbine wakes for the B-pair compared to that for the A-pair. Due to the presence of the downstream turbine preventing a complete wake development, the flow rate through the upstream turbine is reduced, as shown by the reduction of the $\mathrm{x}$ velocity values on U-curve in figure 8 . It should be noted that for the staggered B-pair the $\mathrm{x}$-velocity reduction is observed across the entire width of the upstream turbine, whereas for the side-by-side B-pair the $\mathrm{x}$-velocity reduction is observed only on the inner side of the upstream turbine, as shown earlier in figure 5. As will be shown later, the convergent wake bending of B-pairs will also be responsible for an earlier performance drop for the downstream turbine when the y-distance between the turbine axes is gradually shortened, since the downstream turbine will be in the wake of the upstream turbine more likely for the Bpair than for the A-pair.

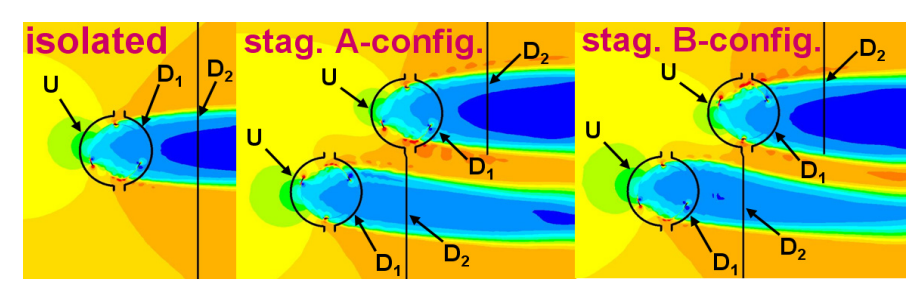

$\mathbf{a}$
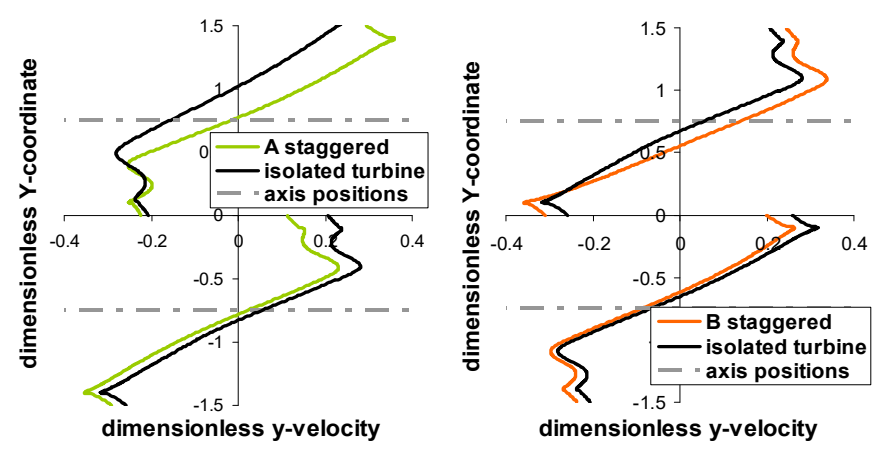

c
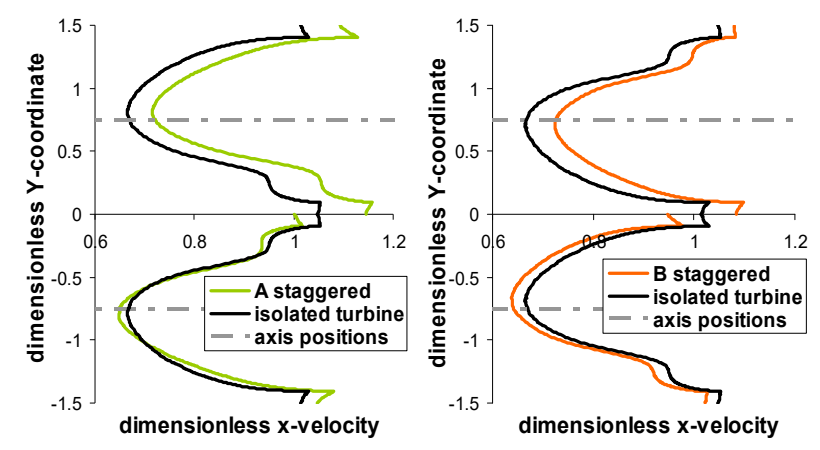

b
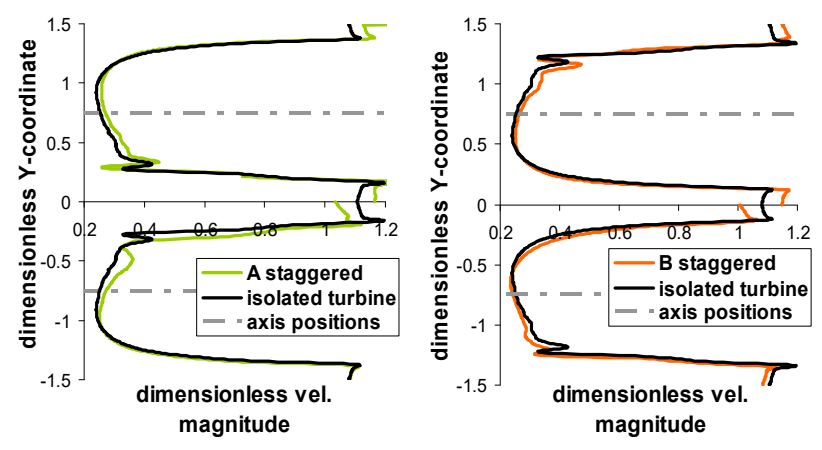

d

FIGURE 8: (a) Velocity magnitude maps $[1 \div 10.5 \mathrm{~m} / \mathrm{s}]$ for the isolated turbine and for the staggered A and B configurations, and the curves set to compare the velocity components; (b) Dimensionless X-velocity on U-curve; (c) Dimensionless y-velocity on U-curve;

(d) Dimensionless velocity magnitude on $\mathrm{D}_{1}$-curve. Results refer to a particular time step of the unsteady solution (blades at $0^{\circ}, 120^{\circ}$ and $240^{\circ}$ azimuthal degrees).

\section{Effect of TSR}


Before discussing the effects of TSR on a turbine pair, the effects on an isolated turbine are briefly illustrated. As can be seen on the velocity magnitude maps in figure 9, an increase in TSR leads to a reduction of the turbine permeability, making the turbine more and more similar to a bluff body (as revealed by the wake shortening and the growth of wake instabilities far downstream).

TSR=2.3

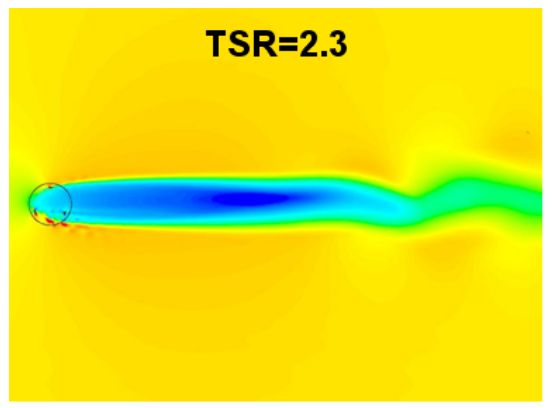

$\boldsymbol{a}$

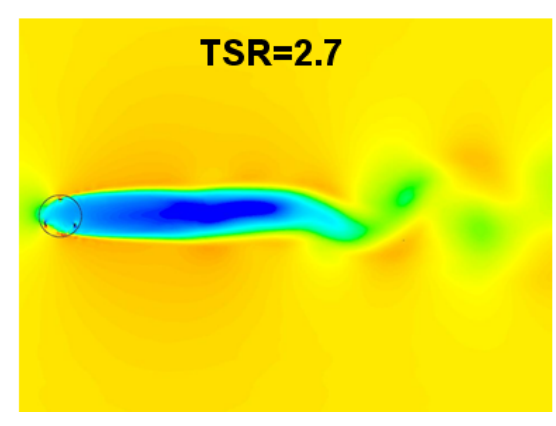

b

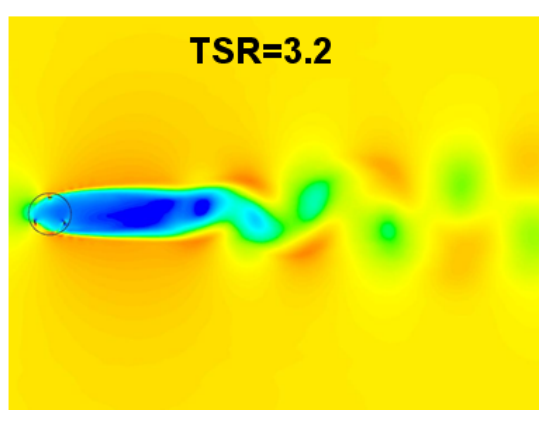

c

FIGURE 9: Velocity magnitude maps $[1 \div 10.5 \mathrm{~m} / \mathrm{s}]$ for the isolated turbine at TSR=2.3 (a), 2.7 (b), 3.2 (c).

The permeability reduction mainly involves two effects observed in the plots of the velocity components upstream the turbine (on U-curve) reported in figure 10: a reduction of the flow rate through the turbine (see the $\mathrm{x}$-velocity decreasing) and an increasing of the flow rate at the turbine sides (see the increase of $x$ - and, especially, of $y$-velocities). As noticeable in the graph of the instantaneous $C_{P}$ in figure 10, the former is responsible for a torque decrement throughout the downwind path of the blade, whereas the latter is responsible for a delay in torque production during the upwind path.

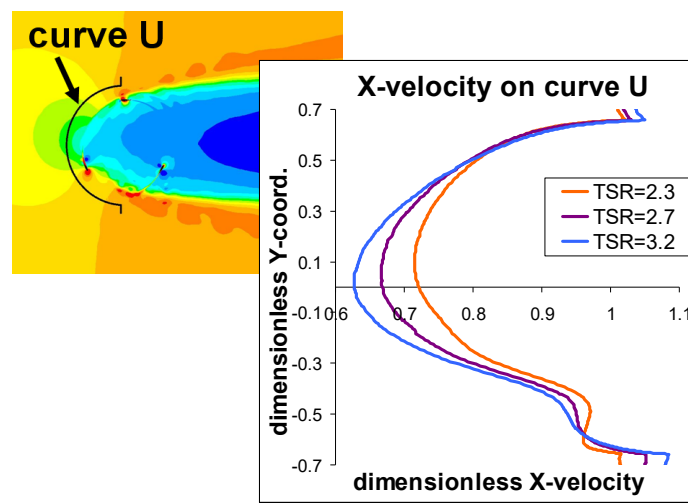

$\mathbf{a}$

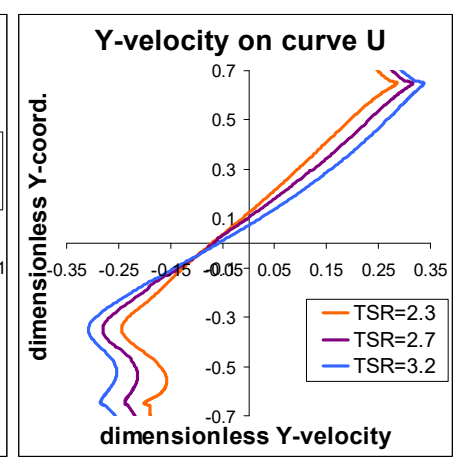

b

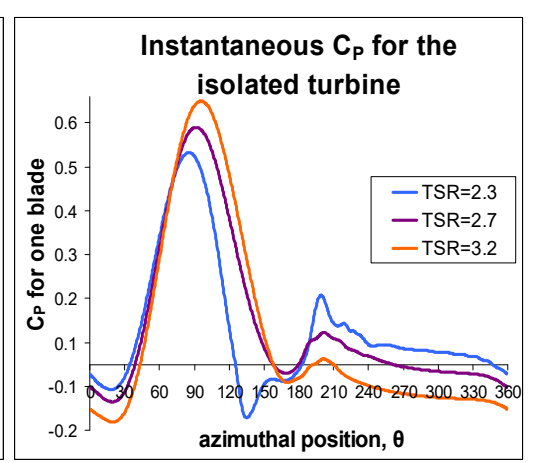

c

FIGURE 10: Dimensionless x-velocity (a) and dimensionless y-velocity (b) calculated on U-curve for the isolated turbine at $\mathrm{TSR}=2.3,2.7,3.2$; (c) one-blade instantaneous $\mathrm{C}_{\mathrm{P}}$ during one revolution, calculated for the isolated turbine at $\mathrm{TSR}=2.3,2.7,3.2$. Results in (a) and (b) refer to a particular time step of the unsteady solution (blades at $0^{\circ}, 120^{\circ}$ and $240^{\circ}$ azimuthal degrees).

It should also be noted that, as already mentioned earlier, the turbine studied here is characterised by a relatively worse performance because of low operational Re that, especially at low TSR (as TSR=2.3), generates flow separation and dynamic stall. Yet, flow separation is moderate at TSR=2.7 and it completely disappears at $\mathrm{TSR}=3.2$; this explains the growth of the $\mathrm{C}_{\mathrm{P}}$ peak value and its occurrence at larger azimuthal angles as the TSR increases. 
Now we look at the effects of TSR on a (non-staggered) pair of turbines. As can be seen from the graph in figure 11, both configurations A and B yield a relative power gain (referring to the turbine spinning at the same TSR) especially at higher TSR. It can also be seen that A-configuration gives a better performance than B-configuration. configuration makes possible a clearer description. To physically explain the increase of power gain obtained (relatively to the isolated turbine) as the TSR increases, we first show that the permeability reduction found for the isolated turbine is even emphasized in case of a pair of turbines. This can be seen from the velocity magnitude maps in figure 12.

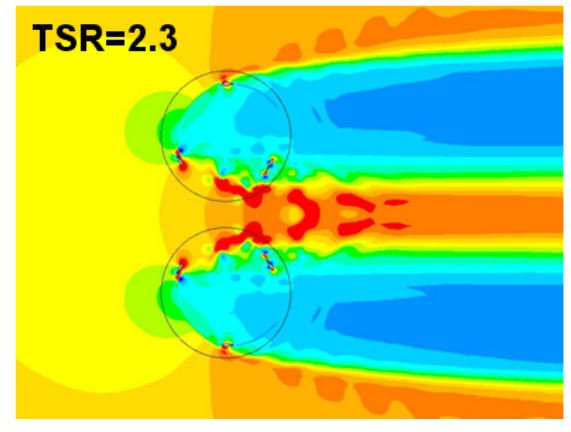

$\mathbf{a}$

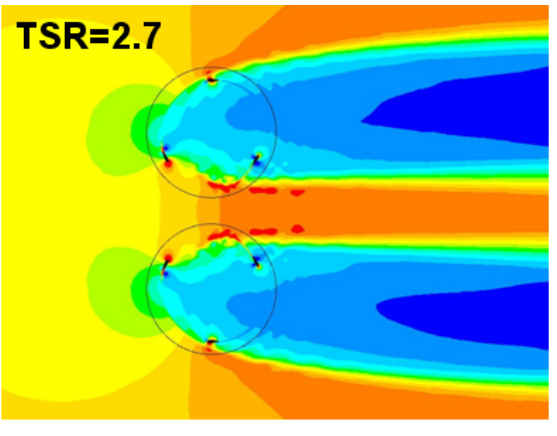

b

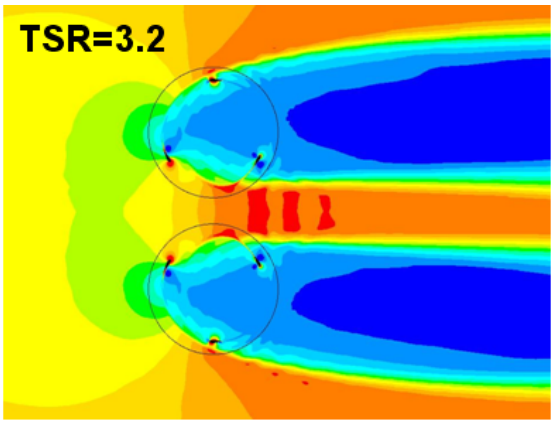

c

FIGURE 12: Velocity magnitude maps $[1 \div 10.5 \mathrm{~m} / \mathrm{s}]$ for A-configuration, calculated at TSR=2.3 (a), 2.7 (b), 3.2 (c).

To further investigate the effects of TSR, $x$ - and y-velocity components upstream of the turbines at $\mathrm{TSR}=2.3,2.7,3.2$ are presented in figure 13. Here we can see that an increase in TSR accentuates three main effects on the interactions between the two turbines. Firstly, as the TSR increases the permeability decreases with respect to the isolated turbine (as recognized by the decrease of $\mathrm{x}$-velocity upstream of the turbines). 
configuration (as recognized by the $\mathrm{x}$ - and y-velocities increasing at the outer sides). Higher values of $\mathrm{y}$ velocity at the outer sides (with respect to the isolated turbine) delay the torque production at the beginning of the upwind path (which means that the torque production starts later as the TSR increases). Thirdly, $a$ drastic reduction of $y$-velocity upstream of the turbines at the inner sides of the configuration occurs as the TSR increases, resulting in a significant extension of torque production during the late part of the upwind path. This last effect seems the main cause for the increase of the relative power gain with TSR, as will be described below.

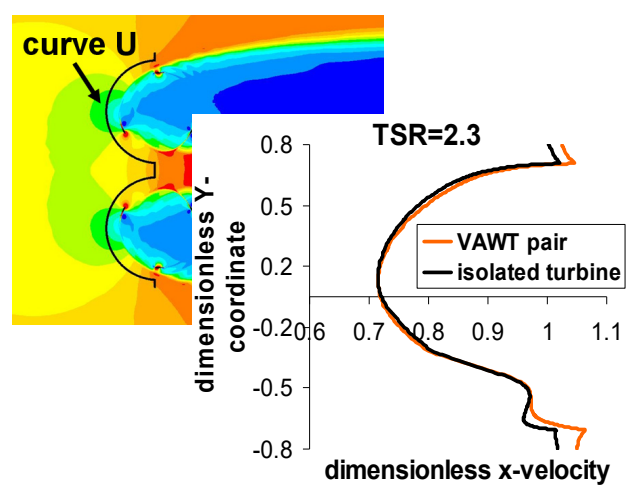

a

$\mathrm{TSR}=2.3$

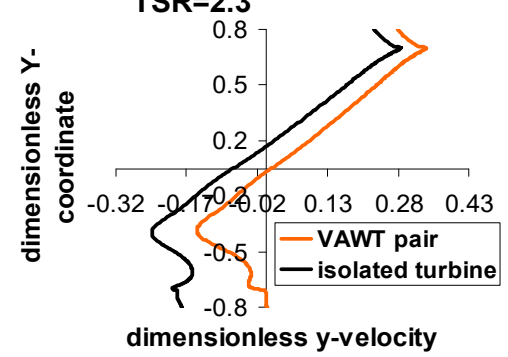

d

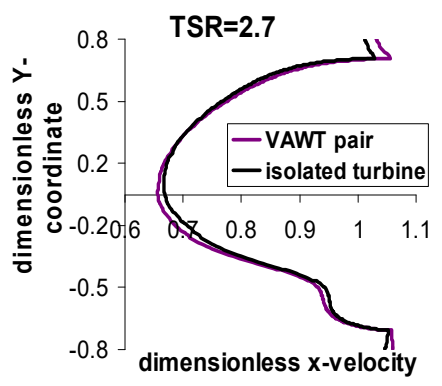

b

TSR=2.7

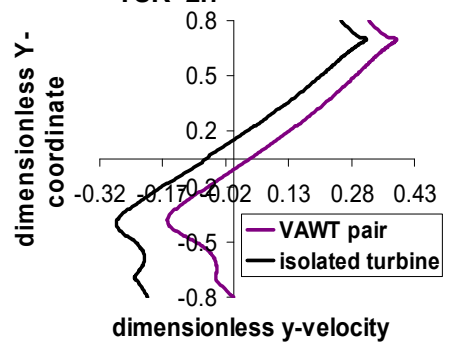

e

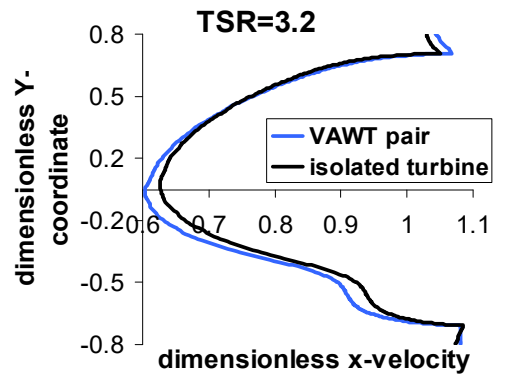

c

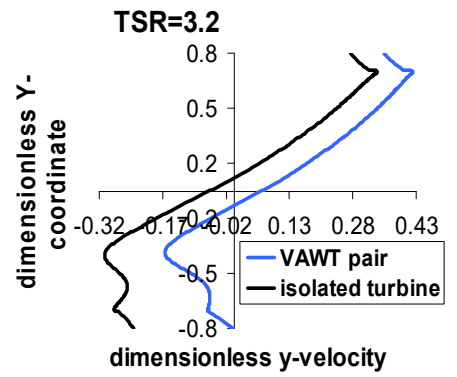

f
362

363

364

365

366

367

368

369

FIGURE 13: Dimensionless x-velocity $(a, b, c)$ and dimensionless y-velocity $(d, e, f)$ calculated at TSR=2.3, 2.7, 3.2 on U-curve for the isolated (anticlockwise spinning) turbine and for the upper (anticlockwise spinning) turbine of A-configuration. Results refer to a particular time step of the unsteady solution (blades at $0^{\circ}, 120^{\circ}$ and $240^{\circ}$ azimuthal degrees).

In figure 14 a comparison of the one-blade instantaneous $C_{P}$ curves for $A$ configuration and for the isolated turbine is presented for three TSR values, together with the percentages of power gains achieved during the upwind and downwind paths.

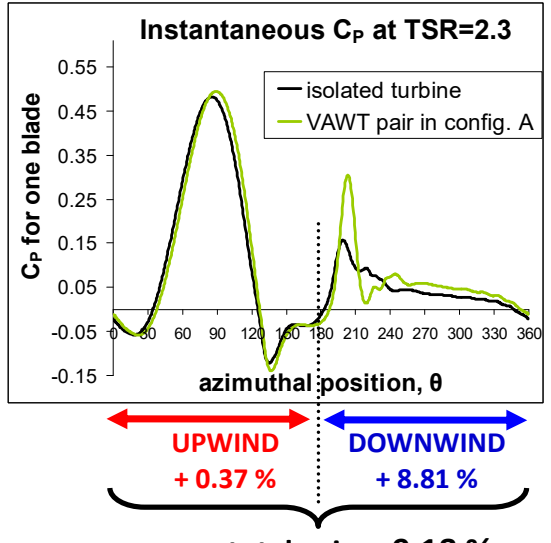

power total gain $+9.18 \%$ with respect to the isolated turbine

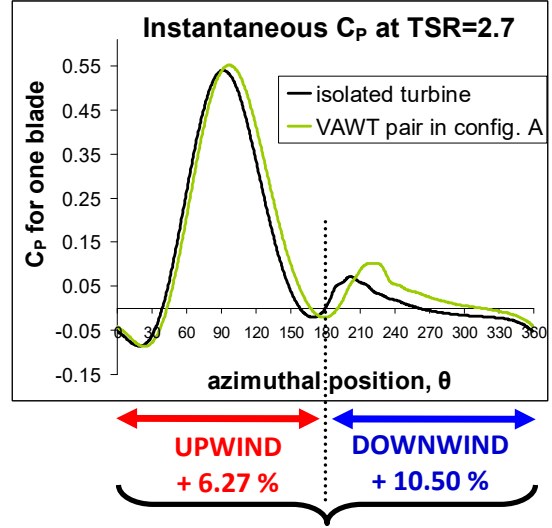

power total gain $+\mathbf{1 6 . 7 8 \%}$ with respect to the isolated turbine

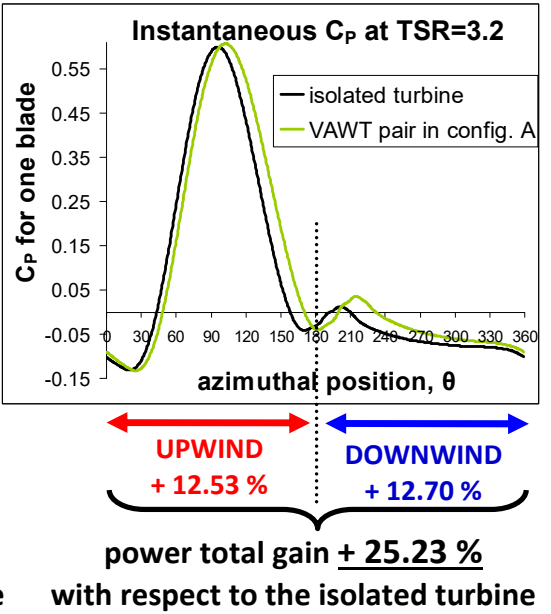


FIGURE 14: One-blade instantaneous $\mathrm{C}_{\mathrm{P}}$ during one revolution calculated for the isolated turbine and for A-configuration at $\mathrm{TSR}=2.3$ (a), 2.7 (b), 3.2 (c); percentages of power gains with respect to the isolated turbine spinning at the same TSR are reported.

It is interesting to observe that the percentage of power gain obtained in the upwind path increases more and more as the TSR increases. However, as also well known from the actuator disk theory, the absolute maximum power is not obtained at the highest TSR since a too high TSR dramatically reduces the flow rate through the turbine, leading to excessively low wind speed in the downwind path (as already seen in the velocity maps in figure 12) and consequently to even negative torque in the downwind path (as noticeable in the one-blade instantaneous $C_{P}$ graphs). Thus the best compromise between the upwind and downwind torque productions is achieved at $\mathrm{TSR}=2.7$, as already shown in figure 11 .

To conclude this section we remark that, although the physical mechanisms responsible for the power increasing in the upwind and downwind paths are expected to be valid for many different types of vertical axis (wind and tidal) turbines, the superiority of one configuration (A or B) and the benefit repartition between the upwind and downwind paths may depend on the turbine solidity and the fluid properties (or the Reynolds number).

\section{Effects of wind direction and distance between turbines}

Wind direction $(\gamma)$ does not affect the power of an isolated VAWT, but does affect the power of a pair of VAWTs. The graph in figure 15 shows the effect of wind direction on the normalised power coefficient $\mathrm{K}$ (defined as the ratio of the turbine's $C_{P}$ to the isolated turbine's $C_{P}$ ) predicted for the anticlockwise (ACW) turbine. Note that this turbine pair can be seen as A-configuration or B-configuration, depending on the wind direction. The distance between the turbine axes is $2 \mathrm{D}$ and TSR is 2.7 for both turbines, which corresponds to the optimal TSR found for a pair of turbines placed side-by-side, whereas the TSR for the reference isolated turbine is 2.55 , which corresponds to the optimal TSR found for the isolated turbine. At $\gamma=270^{\circ}$ the

401 ACW turbine is located directly downstream of the clockwise $(\mathrm{CW})$ turbine; for this wind direction the $\mathrm{K}$ 402 value is not calculated, i.e. we assume the turbine is stopped $\left(\mathrm{C}_{\mathrm{P}}=0\right)$ since the absolute wind speed oncoming the turbine is below the cut-in limit. 


\section{$K$ values for the anticlockwise turbine}

vs. wind direction

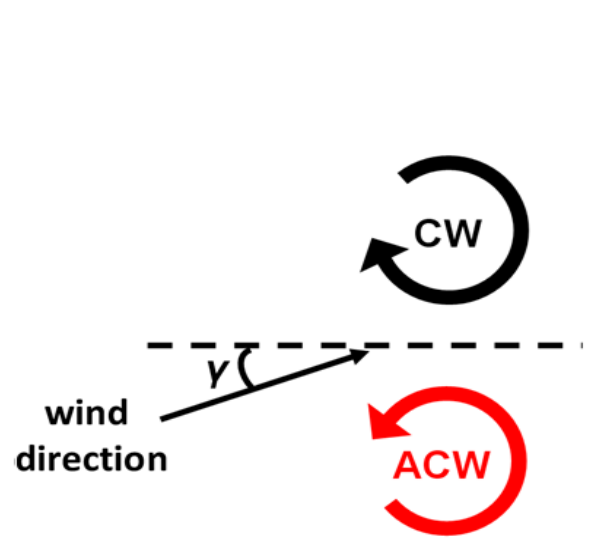

The graph reveals that the turbine performance in the $\gamma$ range $\left[112.5^{\circ}-180^{\circ}-247.5^{\circ}\right]$ is better than in the $\gamma$ 408 range $\left[292.5^{\circ}-0^{\circ}-67.5^{\circ}\right]$. This is related to the difference in the bending of two turbine wakes in these two $\gamma$ 410 ranges, i.e. convergence or divergence of the two wakes, as depicted in figure 16.

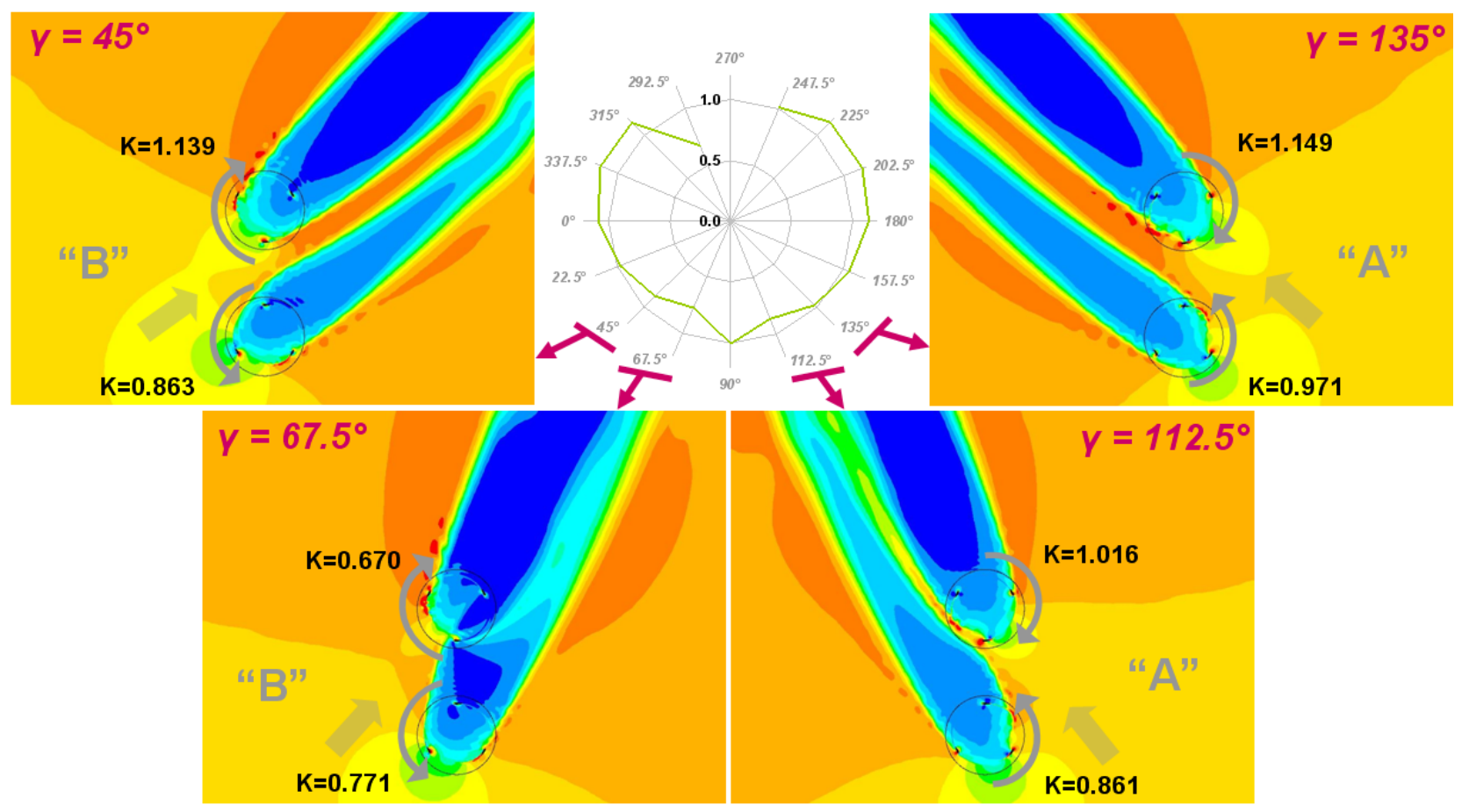

FIGURE 16: normalised power coefficient (K) values and velocity maps in the range $[1 \div 10.5 \mathrm{~m} / \mathrm{s}]$ calculated at $\gamma=45^{\circ}, 67.5^{\circ}, 112.5^{\circ}$, $135^{\circ}$.

From this figure it can be observed that at $\gamma=45^{\circ}$ and $\gamma=67.5^{\circ}$ the turbines work as in the staggered-B configuration, whereas at $\gamma=112.5^{\circ}$ and $\gamma=135^{\circ}$ the turbines work as in the staggered-A configuration. We remark two key findings: (1) the performance of the turbines in A configurations is better than the performance of the turbines in the corresponding B configurations; and (2) with the exception of $\gamma=67.5^{\circ}$, the performance of the downstream turbine is better than the performance of the upstream one. Both these results 
can be explained by the reasons already discussed earlier for the effect of staggering. The poor performance of the upstream turbine at $\gamma=67.5^{\circ}$ (when the convergence of the wakes occurs) is due to the backpressure generated by the downstream turbine that, by preventing a complete development of the wake, causes a reduction of the flow rate through the upstream turbine.

As the upstream turbine is affected by a lower local wind speed, it could be useful to reduce its TSR (for instance, down to 2.55 , which is the optimal value found for the isolated turbine) with keeping the original TSR of 2.7 only for the downstream turbine (except for the cases with $\gamma=0^{\circ}$ and $180^{\circ}$, where the two turbines are side-by-side). The graphs in figure 17 show the effects of the TSR choice on the performance of the ACW turbine and also on the average performance of the two turbines. For completeness the predictions obtained by setting TSR $=2.55$ for both turbines (upstream and downstream) are also presented. The distance between the axes is set to $2 \mathrm{D}$. These results suggest that, for a given wind direction, the best performance is obtained by setting an appropriate TSR for each of the two turbines separately.
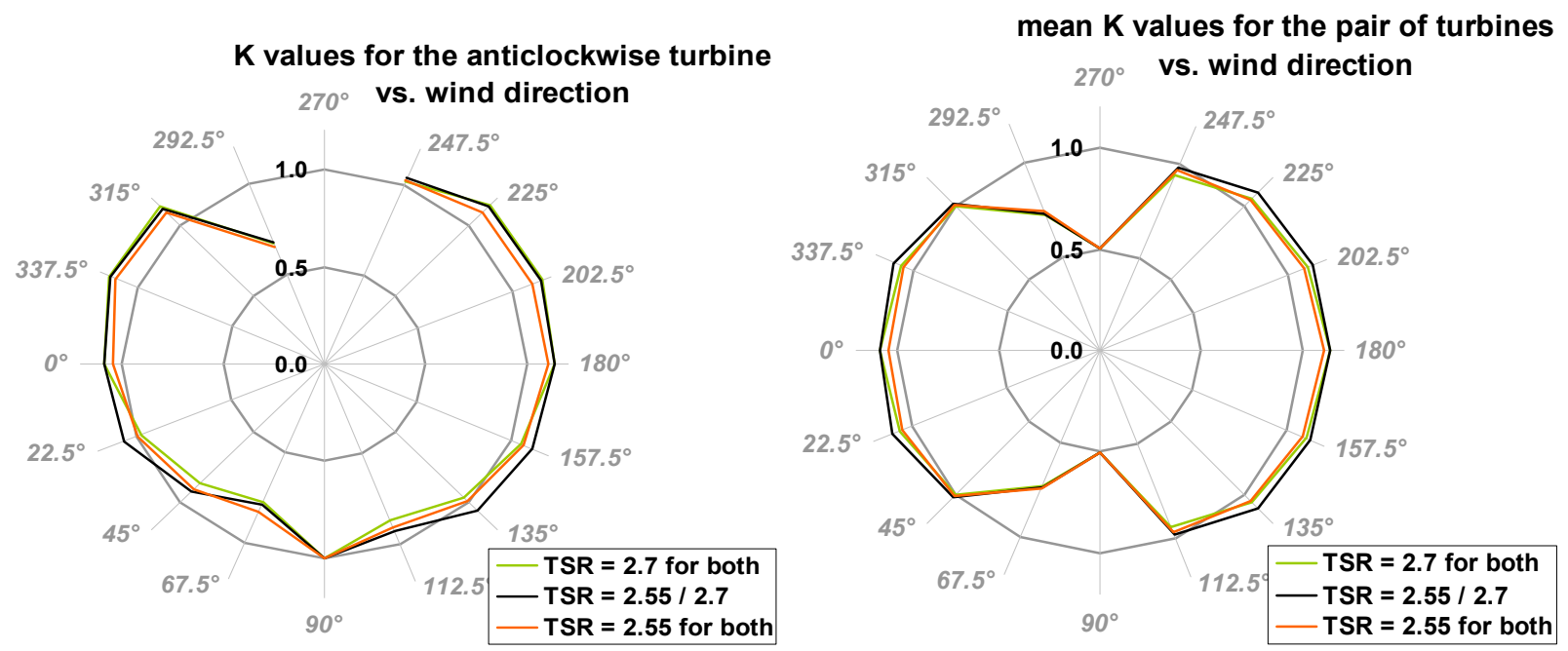

FIGURE 17: Graphs of the normalised power coefficient (K) versus wind direction in case of different rotational speed strategies, for the ACW turbine (left) and averaged of the two turbines (right). Note: the low values of the averaged $\mathrm{K}$ at $\gamma=90^{\circ} / 270^{\circ}$ are due to the assumption that only the upstream turbine is working.

Finally, the graphs in figure 18 illustrate the effects of the distance between the turbine axes on the performance of the ACW turbine and also on the average performance of the two turbines. Four distances are considered: $1.5 \mathrm{D}, 2 \mathrm{D}, 2.5 \mathrm{D}$ and 3D. TSR is set at 2.55 or 2.7 depending on the relative position of each turbine for each wind direction. At short distances (1.5D and 2D) the performance is poor for the wind directions that entail the downstream turbine to be located in the wake of the upstream turbine. This occurs at $\gamma=247.5^{\circ} / 292.5^{\circ}$ for the ACW turbine and, by symmetry, at $\gamma=67.5^{\circ} / 112.5^{\circ} / 247.5^{\circ} / 292.5^{\circ}$ for the overall configuration. Yet for these wind directions the average power loss with respect to the isolated turbine is quite small at longer distances, especially at a distance of 3D. 
$K$ values for the anticlockwise turbine

$270^{\circ}$

vs. wind direction

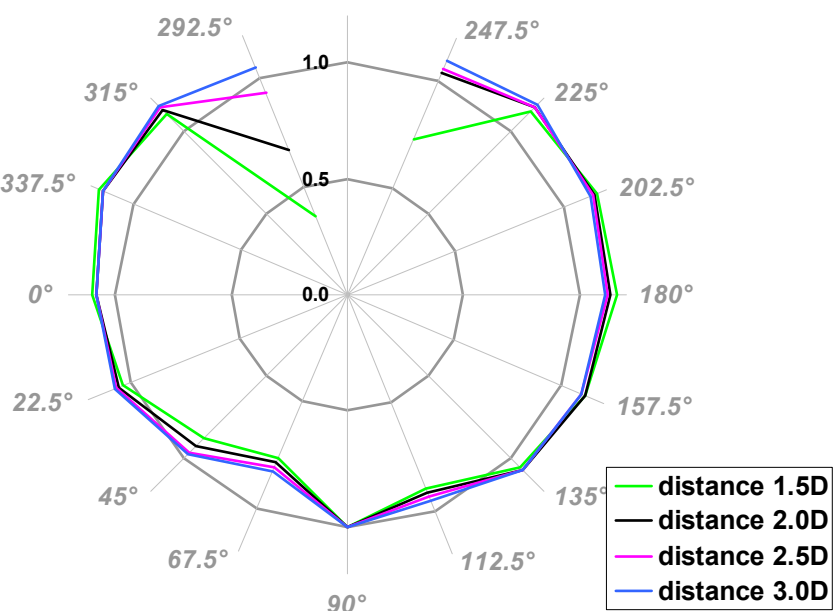

mean $\mathrm{K}$ values for the pair of turbines vs. wind direction

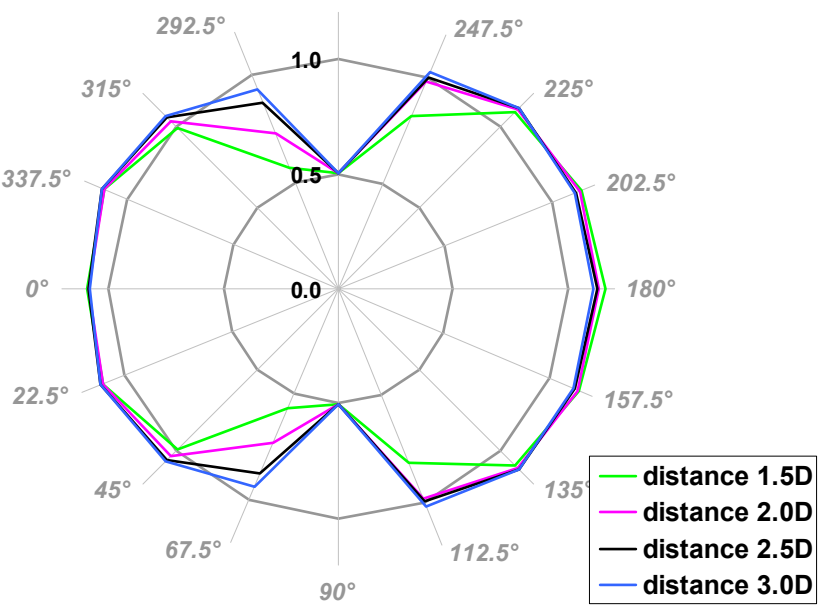

FIGURE 18: Graphs of the normalised power coefficient $(\mathrm{K})$ versus wind direction at different distances between axes, for the ACW turbine (left) and averaged of the two turbines (right). Note: the low values of the averaged $\mathrm{K}$ at $\gamma=90^{\circ} / 270^{\circ}$ are due to the assumption that only the upstream turbine is working.

Interestingly, for the side-by-side situation $\left(\gamma=0^{\circ}\right.$ and $\left.180^{\circ}\right)$ the effect of the turbine distance is much less significant; hence a distance of $3 \mathrm{D}$ appears to be the best overall choice for varying wind directions. It is also important to observe that, although a staggered pair cannot surpass the performance of a side-by-side pair, for wind directions entailing the A-pair situation a distance of 3D yields nearly the same average performance as that for the side-by-side pair for a wide range of $\gamma\left(\right.$ more than $\left.90^{\circ}\right)$.

\section{Discussion}

The 2D CFD analysis performed in this study has explained several important flow mechanisms regarding the performance of a counter-rotating pair of VAWTs. In this section we discuss some implications of the current CFD results for the prediction of the performance of two typical types of VAWT arrays and also the limitations of 2D CFD analysis for each scenario. The two scenarios to be discussed are: (1) a single lateral row of VAWTs with each turbine counter-rotating with respect to neighbouring turbines; and (2) multiple rows (or a farm) of counter-rotating VAWTs.

For the first scenario, we can expect that the performance of such a single lateral row of VAWTs will be explained largely by the flow mechanisms investigated in this study for a pair of counter-rotating turbines. This is because, as long as each turbine in the row is counter-rotating with respect to neighbouring turbines, the local flow field created between any two neighbouring turbines will be similar to either A- or Bconfiguration investigated in this study. One important implication here is that the mechanisms of enhanced power generation by such a single row of VAWTs are a little more complex than the so-called "local blockage effect" explained by the actuator disk theory [13]. As described earlier, the power generated in the upwind path of a VAWT is affected significantly by the local velocity in the lateral (y) direction, which cannot be explained by the $1 \mathrm{D}$ actuator disk theory. It should be noted that the $2 \mathrm{D}$ CFD analysis performed 
476 in this study also has some limitations compared to a full 3D analysis. Presumably the most important 477 limitation is that the recovery rate of turbine wakes predicted by 2D CFD, especially in the far-wake region, 478 is usually lower than a full $3 \mathrm{D}$ case due to the lack of vertical mixing. However, for the case of a single row of VAWTs, we can expect that the details of far-wake mixing will not affect the local flow characteristics around each turbine (except when the wind direction is close to $\gamma=90^{\circ} / 270^{\circ}$, where turbines will be in the wake of other turbines). This means that the local flow mechanisms explained by the current 2D CFD are of direct relevance to the performance of a single row of VAWTs, as long as the aspect ratio of each turbine (the ratio of the blade length to the rotor diameter) is large enough to neglect the blade tip effects.

For the second scenario, where turbines are arrayed not only in the lateral but also in the stream-wise directions to form a VAWT farm, the local flow mechanisms investigated in the current 2D CFD are still expected to be of some importance. The performance of turbines in the most upstream part of the farm may still be explained in a similar manner to the single row case, although that in the downstream part of the farm would be affected by the details of far-wake mixing behind each turbine and also by the reduction of overall flow rate through the entire farm due to the transfer of momentum in the vertical direction, which cannot be predicted by a $2 \mathrm{D}$ analysis. It should be noted that the importance of the local flow mechanisms to the overall performance of the farm is likely to depend on the size of the farm. For a relatively small farm with only a few rows of VAWTs, we can presume that the local flow mechanisms investigated in this study would still be of dominant importance, since the majority of the turbines in the farm would not be significantly affected by the wake of other turbines. For a much larger farm, however, the local flow mechanisms would be of less importance, since the majority of the turbines in the farm would be in the wake of other turbines as well as be influenced by the reduction of overall flow rate through the farm. In such a large farm, the main benefit of employing counter-rotating VAWTs could be that the wake loss is reduced and thus a high-speed flow is maintained throughout the farm, as suggested by Dabiri [2], in analogy with the mechanism of "fish schooling". The recent study by Araya et al. [4] aims to describe approximately the mechanism of this farmpower enhancement using a low-order flow model; however the model is $2 \mathrm{D}$ and is therefore not capable of predicting the reduction of overall flow rate through the farm correctly. Further investigations are required to understand the performance of such a large VAWT farm. counter-rotating VAWTs to identify the local flow mechanisms contributing to their enhanced power generation performance compared to an isolated VAWT. The analysis was performed for two possible configurations of the counter-rotating turbine pair (namely A and B configurations) with various gaps between the two turbines, tip-speed-ratios and wind directions.

For the case of two turbines arrayed side-by-side with respect to the incoming wind (i.e. wind direction $\gamma=0^{\circ} / 180^{\circ}$ ), we have found two key mechanisms contributing to the power increase: (1) change of lateral (y) velocity in the upwind path due to the presence of the neighbouring turbine, making the direction of local 
flow approaching the blade more favourable to generate lift and torque in the upwind path; and (2) contraction of the wake in the downwind path, again due to the presence of the neighbouring turbine, making a larger momentum flux available for power generation in the downwind path. The balance between the two mechanisms (in terms of their contributions to the overall power increase) has been found to depend on the tip-speed-ratio as well as on the configuration type (A or B).

For the case of two turbines arrayed in a staggered pattern with respect to the incoming wind, we have observed that a larger power tends to be generated by the downstream turbine than by the upstream turbine (unless the downstream turbine is in the wake of the upstream turbine). This is essentially due to the upstream turbine blockage, making a high-speed flow available to the downstream turbine. However, the total power of a staggered pair of turbines cannot surpass that of a side-by-side pair of turbines. The total power of a pair of turbines decreases significantly when the wind direction is close to $\gamma=90^{\circ} / 270^{\circ}$, and the value of $\gamma$ at which this significant power decrease occurs depends on the configuration type (A or B). The power tends to remain high for the A-configuration, i.e. when the velocity induced between the two turbines is concordant with the wind direction, since the two turbine wakes in this configuration tend to diverge from each other and hence the downstream turbine is less likely to be in the wake of the upstream turbine.

Finally, we have also discussed some implications of the current 2D CFD results for the prediction of the performance of two typical types of VAWT arrays, namely a single row of counter-rotating VAWTs and multiple rows (or a farm) of counter-rotating VAWTs. For the former case, we can expect that the performance of such a single row of VAWTs will be explained largely by the local flow mechanisms investigated in this study, since the local flow field created between any two neighbouring turbines in such a single row will be similar to either A- or B-configuration studied here. For the latter case, the flow mechanisms investigated in this study are still expected to be of some importance, especially when the farm size is relatively small. As the farm size increases, however, the overall performance of the farm would depend more and more on the details of far-wake mixing of each turbine and also on the reduction of overall flow rate through the farm due to the transfer of momentum in the vertical direction, which cannot be assessed by 2D CFD. Further investigations are therefore required to understand the performance of such a large VAWT farm.

\section{Appendix}

All simulations described in the paper were performed with a reasonably fine grid (grid (1)); $y^{+}$is less than 3 except for the trailing edge, where few elements with $y^{+} \sim 5$ appear due to the difficulty to generate regular and small quad elements on a sharp trailing edge. To investigate the grid sensitivity, some simulations are repeated with a new grid (grid (2)) employing a rounded trailing edge, with a radius of $0.5 \%$ of the chord length, allowing the regular growing of quad elements all around the trailing edge, and a smaller wall distance from the first layer of cells, resulting in $y^{+}<0.5$ all around the blade. An additional finer grid (grid (3)), characterised by a greater number of elements on the blade profile and on the interface between 


\begin{tabular}{|c|c|c|c|c|c|c|}
\hline $\begin{array}{l}\text { Case } \\
\text { name }\end{array}$ & $y^{+}$ & $\begin{array}{l}\text { Nodes on } \\
\text { blade } \\
\text { profile }\end{array}$ & $\begin{array}{l}\text { Nodes on } \\
\text { rotating } \\
\text { interface }\end{array}$ & $\begin{array}{l}\text { Cells in each } \\
\text { rotating domain }\end{array}$ & $\begin{array}{l}\text { Total domain cells } \\
\text { for the isolated } \\
\text { turbine case }\end{array}$ & $\begin{array}{l}\text { Total domain } \\
\text { cells for the } \\
\text { turbine pair case }\end{array}$ \\
\hline Grid (1) & $<3$ & 440 & 720 & 117000 & 246000 & 383000 \\
\hline Grid (2) & $<0.5$ & 440 & 720 & 131000 & 260000 & 411000 \\
\hline Grid (3) & $<0.5$ & 700 & 1200 & 246000 & 385000 & 666000 \\
\hline
\end{tabular}

553

554

555

556

557

558

559

560

561

562

563
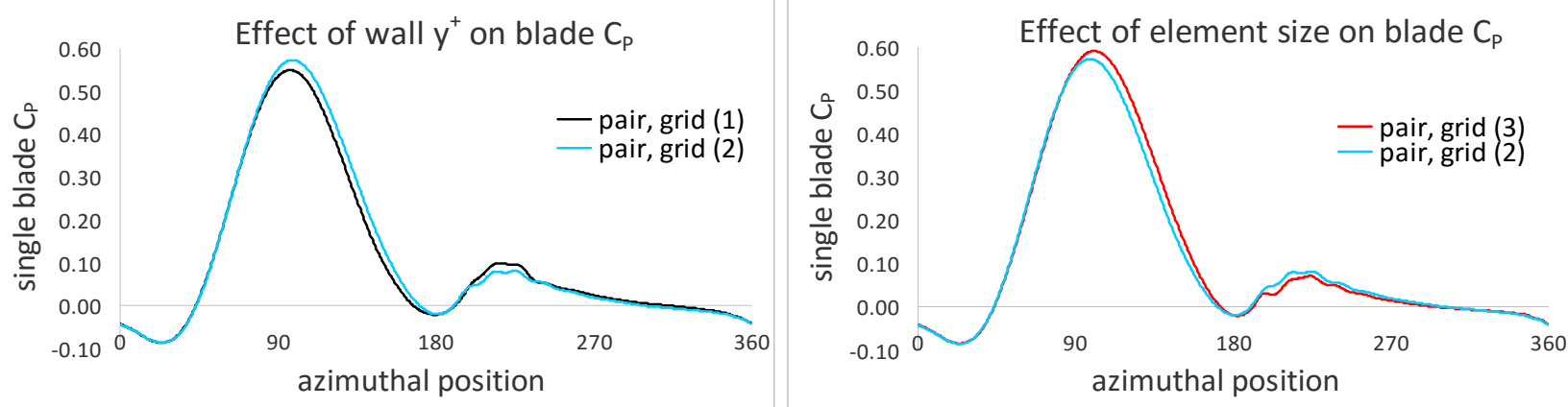

FIGURE 20: Effect of grid refinement on the instantaneous one blade $C_{P}$ in the case of A-pair.

\begin{tabular}{|c|c|c|c|c|}
\hline $\begin{array}{c}\text { Case } \\
\text { name }\end{array}$ & $\begin{array}{c}\mathrm{C}_{\mathrm{P}} \\
\text { isolated } \\
\text { turbine }\end{array}$ & $\begin{array}{c}\mathrm{C}_{\mathrm{P}} \\
\text { pair }\end{array}$ & $\begin{array}{c}\text { power ratio: } \\
\mathrm{C}_{\mathrm{P}, \text { pair }} / \mathrm{C}_{\mathrm{P}, \text { isolated }}\end{array}$ & $\begin{array}{c}(\text { power ratio)-(power ratio) grid(1) }) /(\text { power } \\
\text { ratio) grid(1) }\end{array}$ \\
\hline Grid (1) & 0.274 & 0.321 & 1.171 & - \\
\hline Grid (2) & 0.287 & 0.336 & 1.172 & $0 \%$ \\
\hline Grid (3) & 0.300 & 0.348 & 1.160 & $-0.94 \%$ \\
\hline
\end{tabular}

TABLE 3: Main results of the grid sensitivity study.
Effect of element size on blade $C_{p}$

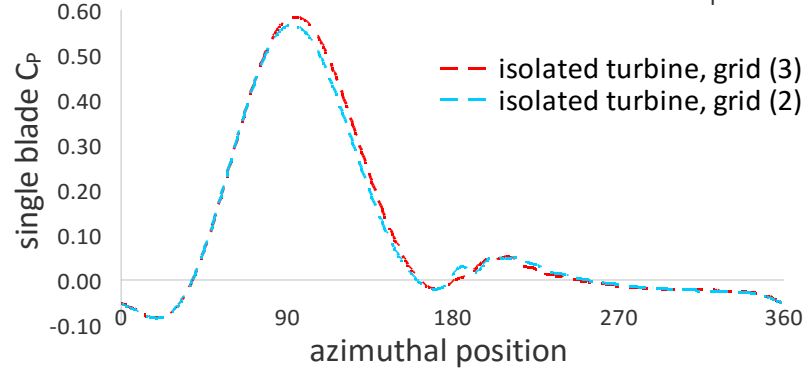

FIGURE 19: Effect of grid refinement on the instantaneous one blade $C_{P}$ in the case of isolated turbine. 
Table 3 summarises the turbine performance obtained in terms of the absolute $C_{P}$ and of the "power ratio", i.e. normalised power gain for the A-pair case with respect to the isolated turbine case. The results obtained with the grid (3) are considered to be more accurate in terms of the absolute turbine performance, but require much more computational resources than the grids (1) and (2). Yet, the most important conclusion from this grid sensitivity study is that, despite the non-negligible effects of $y^{+}$and element size on the absolute turbine performance, there are no significant effects on the power gain for the turbine pair with respect to the isolated turbine. Since the main focus of the present paper is on the behaviour of a pair of turbines compared to the behaviour of the isolated turbine, even grid (1) can be considered sufficiently accurate.

\section{References}

[1] Whittlesey R. W., Liska S. and Dabiri J. O., "Fish schooling as a basis for vertical axis wind turbine farm design", Bioinspiration \& biomimetics, 2010, vol.5

[2] Dabiri J. O., "Potential order-of-magnitude enhancement of wind farm power density via counter-rotating vertical-axis wind turbine arrays", Journal of renewable and sustainable energy, 2011, vol. 3

[3] Kinzel M., Mulligan Q. and Dabiri J. O., "Energy exchange in an array of vertical-axis wind turbines", Journal of Turbulence, 2012, vol. 13, No. 38, pp. 1-13

[4] Feng G. et al. "Optimizing the Land Use for Wind Farms Using Vertical Axis Wind Turbines”, PO.ID 192, Europe's premier wind energy conference and exhibition, European Wind Energy Association (EWEA), 2014, Barcelona, Spain.

[5] Araya D. B., Craig, A. E., Kinzel, M., Dabiri, J. O., "Low-order modeling of wind farm aerodynamics using leaky Rankine bodies", Journal of renewable and sustainable energy, 2014, vol. 6, 063118.

[6] F. Balduzzi, A. Bianchini, R. Maleci, G. Ferrara, L. Ferrari, Critical issues in the CFD simulation of Darrieus wind turbines, Renewable Energy 85 (2016) 419-435.

[7] Windward Engineering. Power Performance Test Report for Windspire, 2013, http://windwardengineering.com/ourwork/projects/windspire/windspire-rtc-testing/

[8] Ansys Fluent 12.0. User's Guide, Ansys Inc. (2009).

[9] F. R. Menter, "Zonal two equation k-omega model for aerodynamic flows", in: 24th Fluid Dynamics Conference, July 6-9, Orlando, Florida, 1993.

[10] Nobile R, Vahdati M, Barlow JF, Mewburn-Crook A. "Unsteady flow simulation of a vertical axis augmented wind turbine: A two-dimensional study”, J. Wind Eng. Ind. Aerodyn.125 (2014) 168-179.

600

[11] M. C. Claessens, “The Design and Testing of Airfoils for Application in Small Vertical Axis Wind Turbines”, Master of Science

602 [12] S. Giorgetti, G. Pellegrini, S. Zanforlin, "CFD investigation on the aerodynamic interferences between medium-solidity Darrieus 603 vertical axis wind turbines", Energy Procedia 81 (2015) 227-239.

604 [13] Nishino T., Draper S., “Local blockage effect for wind turbines”, Journal of Physics: Conference Series, 2015, vol. 625, 012010. 